hep-th/0309105

\title{
Two Coupled Harmonic Oscillators on Non-commutative Plane
}

\author{
Ahmed Jellal $^{1 *}$, El Hassan El Kinani ${ }^{2,3 \dagger}$ and Michael Schreiber ${ }^{1}$ \\ ${ }^{1}$ Institut für Physik, Technische Universität, \\ D-09107 Chemnitz, Germany \\ 2 The Abdus Salam International Center for Theoretical Physics, \\ Strada Costiera 11, 34014 Trieste, Italy \\ ${ }^{3}$ Moulay Ismail University, Faculty of Science and Technical \\ Mathematics Department, P.O. Box 509, Boutalamine, Errachidia, Morocco
}

\begin{abstract}
We investigate a system of two coupled harmonic oscillators on the non-commutative plane $\mathbb{R}_{\theta}^{2}$ by requiring that the spatial coordinates do not commute. We show that the system can be diagonalized by a suitable transformation, i.e. a rotation with a mixing angle $\alpha$. The obtained eigenstates as well as the eigenvalues depend on the non-commutativity parameter $\theta$. Focusing on the ground state wave function before the transformation, we calculate the density matrix $\rho_{0}(\theta)$ and find that its traces $\operatorname{Tr}\left(\rho_{0}(\theta)\right)$ and $\operatorname{Tr}\left(\rho_{0}^{2}(\theta)\right)$ are not affected by the non-commutativity. Evaluating the Wigner function on $\mathbb{R}_{\theta}^{2}$ confirms this. The uncertainty relation is explicitly determined and found to depend on $\theta$. For small values of $\theta$, the relation is shifted by a $\theta^{2}$ term, which can be interpreted as a quantum correction. The calculated entropy does not change with respect to the normal case. We consider the limits $\alpha=0$ and $\alpha=\frac{\pi}{2}$. In first case, by identifying $\theta$ to the squared magnetic length, one can recover basic features of the Hall system.
\end{abstract}

\footnotetext{
*E-mail: jellal@gursey.gov.tr

${ }^{\dagger}$ E-mail: hkinani@ictp.trieste.it
} 


\section{Introduction}

Because of its mathematical simplicity, the harmonic oscillator provides solvable models in many branches of physics. It often gives a clear illustration of an abstract idea. For instance a charged particle on the plane $\vec{x}=\left(x_{1}, x_{2}\right)$ in presence of a strong uniform magnetic field described by the Lagrangian

$$
L=\frac{m}{2} \dot{\vec{r}}^{2}+\vec{A} \cdot \dot{\vec{x}}-V(\vec{x})
$$

where the vector potential $\vec{A}=\left(A_{1}, A_{2}\right)$ and the confining potential are

$$
A_{i}=\frac{1}{2} B \epsilon_{i j} x_{j}, \quad V(\vec{x})=\frac{1}{2} \omega \vec{x}^{2}
$$

with $\epsilon_{12}=-\epsilon_{21}=1$ and 0 otherwise, gives a nice natural non-commutative system [1] and also a good starting point to discuss the quantum Hall effect [2]. Now it is natural to ask what happens if the external potential is not parabolic? This question was answered by Kim et al. $[3-11]$ starting more than twenty years ago. They considered two coupled harmonic oscillators with the potential

$$
V(\vec{x})=\frac{1}{2}\left(c_{1} x_{1}^{2}+c_{2} x_{2}^{2}+c_{3} x_{1} x_{2}\right)
$$

with constant $c_{1}, c_{2}, c_{3}$ and explicitly determined the corresponding density matrix and the Wigner function as well as other quantities.

There are many physical models based on coupled harmonic oscillators, such as the Lee model in quantum field theory [12, the Bogoliubov transformation in superconductivity 13 , two-mode squeezed states of light [8, 14, 15, the covariant harmonic oscillator model for the parton picture [6], and models in molecular physics [16]. There are also models of current interest in which one of the variables is not observed, including thermo-field dynamics [17, twomode squeezed states [18, 19, the hadronic temperature [5], and the Barnet-Phoenix version of information theory [20. In all of these cases, the mixing angle $\alpha$ of the employed transformation is $\frac{\pi}{2}$, and in this situation the mathematics becomes simple.

In this paper we study quantum mechanically a system of two coupled harmonic oscillators

on the non-commutative (NC) plane $\mathbb{R}_{\theta}^{2}$. This can be done by demanding that the spatial coordinates do not commute. We use the star-product to write the NC Hamiltonian and solve the eigenequations to get the eigenstates as well as the energy spectrum. Focusing on the ground state, we evaluate the corresponding density matrix. It depends on the non-commutativity parameter $\theta$ but its traces as well as the entropy are not affected by the non-commutativity. Also we calculate the Wigner function to confirm the $\theta$-independence of the traces. The uncertainty relation on $\mathbb{R}_{\theta}^{2}$ is found to depend on $\theta$ and it coincides with the normal case in the limit $\theta=0$. 
For small $\theta$, we show that this relation contains a quantum correction, which is a shift with a $\theta^{2}$ term. Also we discuss some limits of the mixing angle, namely $\alpha=\frac{\pi}{2}$ and $\alpha=0$. In the last case, the system can be linked to the Hall electron.

In section 2, we give the energy spectrum and the eigenstates of a Hamiltonian describing two coupled harmonic oscillators. This serves as a guide in section 3 where we consider the same system but require that the spatial coordinates do not commute. In section 4, we deal with the corresponding density matrix and evaluate its traces. In section 5 we calculate the Wigner function on $\mathbb{R}_{\theta}^{2}$ and investigate its link with the density matrix. We determine explicitly the uncertainty relation as well as the entropy in section 6. Some particular cases will be considered in section 7. Finally, we conclude our work in the last section.

\section{Coupled harmonic oscillators}

Let us consider a system of two coupled harmonic oscillators parameterized by the coordinates $X_{1}, X_{2}$ and masses $m_{1}, m_{2}$. This can be described by a Hamiltonian as the sum of free and interacting parts [1]

$$
H_{1}=\frac{1}{2 m_{1}} P_{1}^{2}+\frac{1}{2 m_{2}} P_{2}^{2}+\frac{1}{2}\left(C_{1} X_{1}^{2}+C_{2} X_{2}^{2}+C_{3} X_{1} X_{2}\right)
$$

where $C_{1}, C_{2}, C_{3}$ are constant parameters. After rescaling the position variables

$$
x_{1}=\left(m_{1} / m_{2}\right)^{\frac{1}{4}} X_{1}, \quad x_{2}=\left(m_{2} / m_{1}\right)^{\frac{1}{4}} X_{2}
$$

as well as the momentum

$$
p_{1}=\left(m_{2} / m_{1}\right)^{\frac{1}{4}} P_{1}, \quad p_{2}=\left(m_{1} / m_{2}\right)^{\frac{1}{4}} P_{2}
$$

$H_{1}$ can be written as

$$
H_{2}=\frac{1}{2 m}\left(p_{1}^{2}+p_{2}^{2}\right)+\frac{1}{2}\left(c_{1} x_{1}^{2}+c_{2} x_{2}^{2}+c_{3} x_{1} x_{2}\right)
$$

where the parameters are

$$
m=\left(m_{1} m_{2}\right)^{1 / 2}, \quad c_{1}=C_{1} \sqrt{\frac{m_{2}}{m_{1}}}, \quad c_{1}=C_{1} \sqrt{\frac{m_{1}}{m_{2}}}, \quad c_{3}=C_{3} .
$$

As the Hamiltonian (7) involves an interacting term, a straightforward investigation of the basic features of the system is not easy. Nevertheless, we can simplify this situation by a transformation to new phase space variables

$$
y_{a}=M_{a b} x_{b}, \quad q_{a}=M_{a b} p_{b}
$$


where the matrix

$$
\left(M_{a b}\right)=\left(\begin{array}{cc}
\cos \frac{\alpha}{2} & -\sin \frac{\alpha}{2} \\
\sin \frac{\alpha}{2} & \cos \frac{\alpha}{2}
\end{array}\right)
$$

is a unitary rotation with the mixing angle $\alpha$. Inserting the mapping (9) into (77), one realizes that $\alpha$ should satisfy the condition

$$
\tan \alpha=\frac{c_{3}}{c_{2}-c_{1}}
$$

to get a factorizing Hamiltonian

$$
H_{3}=\frac{1}{2 m}\left(q_{1}^{2}+q_{2}^{2}\right)+\frac{K}{2}\left(e^{2 \eta} y_{1}^{2}+e^{-2 \eta} y_{2}^{2}\right)
$$

where

$$
K=\sqrt{c_{1} c_{2}-c_{3}^{2} / 4}, \quad e^{\eta}=\frac{c_{1}+c_{2}+\sqrt{\left(c_{1}-c_{2}\right)^{2}+c_{3}^{2}}}{2 K}
$$

and the condition $4 c_{1} c_{2}>c_{3}^{2}$ must be fulfilled.

It is convenient to separate (12) into two commuting parts

$$
H_{3}=e^{\eta} \mathcal{H}_{1}+e^{-\eta} \mathcal{H}_{2}
$$

where $\mathcal{H}_{1}$ and $\mathcal{H}_{2}$ are given by

$$
\mathcal{H}_{1}=\frac{1}{2 m} e^{-\eta} q_{1}^{2}+\frac{K}{2} e^{\eta} y_{1}^{2}, \quad \mathcal{H}_{2}=\frac{1}{2 m} e^{\eta} q_{2}^{2}+\frac{K}{2} e^{-\eta} y_{2}^{2} .
$$

First, one can see that the decoupled Hamiltonian

$$
H_{0}=\frac{1}{2 m} q_{1}^{2}+\frac{K}{2} y_{1}^{2}+\frac{1}{2 m} q_{2}^{2}+\frac{K}{2} y_{2}^{2}
$$

is obtained for $\eta=0$. Second, it is interesting to note that (16) can be derived by a canonical transformation only from

$$
\mathcal{H}=\mathcal{H}_{1}+\mathcal{H}_{2}
$$

rather than from (14). This suggests that it might be advantageous to consider (17) instead of (14). Because of that Kim et al. [10,11] were focusing on the Hamiltonian (17).

It is clear that $\mathcal{H}$ is a Hamiltonian of two decoupled harmonic oscillators. Thus it can simply be diagonalized by defining a set of creation and annihilation operators

$$
a_{i}=\sqrt{\frac{K}{2 \hbar \omega}} e^{\frac{\eta}{2}} y_{i}+\frac{i}{\sqrt{2 m \hbar \omega}} e^{-\frac{\eta}{2}} q_{i}, \quad a_{i}^{\dagger}=\sqrt{\frac{K}{2 \hbar \omega}} e^{\frac{\eta}{2}} y_{i}-\frac{i}{\sqrt{2 m \hbar \omega}} e^{-\frac{\eta}{2}} q_{i}
$$

with frequency

$$
\omega=\sqrt{\frac{K}{m}}
$$


They satisfy the commutation relations

$$
\left[a_{i}, a_{j}^{\dagger}\right]=\delta_{i j}
$$

Other commutators vanish. Now we can map $\mathcal{H}$ in terms of $a_{i}$ and $a_{i}^{\dagger}$ as

$$
\mathcal{H}=\hbar \omega\left(a_{1}^{\dagger} a_{1}+a_{2}^{\dagger} a_{2}+1\right)
$$

To obtain the eigenstates and the eigenvalues, one solves the eigenequations

$$
\mathcal{H}\left|n_{1}, n_{2}\right\rangle=\mathcal{E}_{n_{1}, n_{2}}\left|n_{1}, n_{2}\right\rangle
$$

getting

$$
\left|n_{1}, n_{2}\right\rangle=\frac{\left(a_{1}^{\dagger}\right)^{n_{1}}\left(a_{2}^{\dagger}\right)^{n_{2}}}{\sqrt{n_{1} ! n_{2} !}}|0,0\rangle
$$

where the ground state wave function is

$$
\psi_{0}(\vec{y}) \equiv\left\langle y_{1}, y_{2} \mid 0,0\right\rangle=\sqrt{\frac{m \omega}{\pi \hbar}} \exp \left\{-\frac{m \omega}{2 \hbar}\left(e^{\eta} y_{1}^{2}+e^{-\eta} y_{2}^{2}\right)\right\}
$$

as well as the energy spectrum

$$
\mathcal{E}_{n_{1}, n_{2}}=\hbar \omega\left(n_{1}+n_{2}+1\right)
$$

The above solutions can be used to deduce those corresponding to $H_{3}$, in particular its energy spectrum

$$
E_{3, n_{1}, n_{2}}=\hbar \omega\left(e^{\eta}\left(n_{1}+\frac{1}{2}\right)+e^{-\eta}\left(n_{2}+\frac{1}{2}\right)\right)
$$

and the ground state wave function

$$
\begin{aligned}
\psi_{0}(\vec{x}) & \equiv\left\langle x_{1}, x_{2} \mid 0,0\right\rangle \\
& =\sqrt{\frac{m \omega}{\pi \hbar}} \exp \left\{-\frac{m \omega}{2 \hbar}\left[e^{\eta}\left(x_{1} \cos \frac{\alpha}{2}-x_{2} \sin \frac{\alpha}{2}\right)^{2}+e^{-\eta}\left(x_{1} \sin \frac{\alpha}{2}+x_{2} \cos \frac{\alpha}{2}\right)^{2}\right]\right\} .
\end{aligned}
$$

While (24) is separable in terms of the variables $y_{1}$ and $y_{2}$, this is not the case for (27) in terms of $x_{1}$ and $x_{2}$.

In what follows, we generalize the present system to the NC case by deforming the spatial configuration. This will be used to investigate some physical quantities of the system, i.e. the density matrix and the Wigner function as well as other quantities corresponding to the ground state wave function (27) in the NC case. 


\section{Non-commutative system}

We proceed by using the NC geometry [21] to study two coupled harmonic oscillators. We demand that the coordinates of the plane do not commute

$$
\left[x_{i}, x_{j}\right]=i \theta_{i j}
$$

where $\theta_{i j}=\epsilon_{i j} \theta$ is the non-commutativity parameter. This relation can be obtained using the star-product definition

$$
f(x) \star g(x)=\left.\exp \left\{\frac{i}{2} \theta_{i j} \partial_{x^{i}} \partial_{y^{j}}\right\} f(x) g(y)\right|_{x=y}
$$

where $f$ and $g$ are two arbitrary functions, supposed to be infinitely differentiable. In what follows, we will use the standard commutation relations

$$
\left[p_{i}, x_{j}\right]=-i \delta_{i j}, \quad\left[p_{i}, p_{j}\right]=0
$$

supplemented by the relation (28). Together they define a generalized quantum mechanics, which leads to the standard one for $\theta=0$.

Now let us define the Hamiltonian (17) on $\mathbb{R}_{\theta}^{2}$. Noting that $H_{2}$ acts on an arbitrary function $\Psi(\vec{r}, t)$ as

$$
H_{2} \star \Psi(\vec{r}, t)=H_{2}^{\mathrm{nc}} \Psi(\vec{r}, t)
$$

and applying the definition (29) we obtain

$$
H_{2}^{\mathrm{nc}}=\frac{1}{2 m}\left(p_{1}^{2}+p_{2}^{2}\right)+\frac{c_{1}}{2}\left(x_{1}-\frac{\theta}{2 \hbar} p_{2}\right)^{2}+\frac{c_{2}}{2}\left(x_{2}+\frac{\theta}{2 \hbar} p_{1}\right)^{2}+\frac{c_{3}}{2}\left(x_{1}-\frac{\theta}{2 \hbar} p_{2}\right)\left(x_{2}+\frac{\theta}{2 \hbar} p_{1}\right) .
$$

With (32), we actually have two possibilities to get the NC version of (17). This can be done either by transforming $H_{2}^{\text {nc }}$ via (9) to obtain

$$
\mathcal{H}^{\mathrm{nc}}=\frac{1}{2 M}\left(e^{-\eta} q_{1}^{2}+e^{\eta} q_{2}^{2}\right)+\frac{K}{2}\left(e^{\eta} y_{1}^{2}+e^{-\eta} y_{2}^{2}\right)+\frac{K \theta}{2 \hbar}\left(e^{-\eta} y_{2} q_{1}-e^{\eta} y_{1} q_{2}\right)
$$

or by starting straightforwardly from (17), using (29) to end up with (33). The effective mass is given by

$$
M=\frac{m}{1+\left(\frac{m \omega \theta}{2 \hbar}\right)^{2}} .
$$

It is useful to write (33) in the compact form

$$
\mathcal{H}^{\prime \text { nc }}=\frac{1}{2 M}\left(Q_{1}^{2}+Q_{2}^{2}\right)+\frac{K}{2}\left(Y_{1}^{2}+Y_{2}^{2}\right)+\frac{K \theta}{2 \hbar}\left(Y_{2} Q_{1}-Y_{1} Q_{2}\right)
$$


by rescaling the variables to new coordinates $Y_{i}$ and $Q_{i}$. Comparing $\mathcal{H}^{\prime \text { nc }}$ to $\mathcal{H}$, we note that $\mathcal{H}^{\prime} \mathrm{nc}$ contains an additional term proportional to $\theta$ which is basically the angular momentum. Also, the NC system shows an effective mass $M$ which coincides with the mass $m$ for $\theta=0$.

For the diagonalization of $\mathcal{H}^{\prime n c}$ we express the position and momentum variables in terms of creation and annihilation operators

$$
Y_{i}=\sqrt{\frac{\hbar \Omega}{2 K}}\left(b_{i}+b_{i}^{\dagger}\right), \quad Q_{i}=i \sqrt{\frac{M \hbar \Omega}{2}}\left(b_{i}^{\dagger}-b_{i}\right)
$$

which commute and satisfy the relations

$$
\left[b_{i}, b_{j}^{\dagger}\right]=\delta_{i j}
$$

where the effective frequency

$$
\Omega=\sqrt{\frac{K}{M}}
$$

depends on $\theta$. With the help of another set of operators

$$
\begin{aligned}
B_{1} & =\frac{1}{\sqrt{2}}\left(b_{1}+i b_{2}\right), & & B_{1}^{\dagger}=\frac{1}{\sqrt{2}}\left(b_{1}^{\dagger}-i b_{2}^{\dagger}\right) \\
B_{2} & =\frac{1}{\sqrt{2}}\left(-b_{1}+i b_{2}\right), & B_{2}^{\dagger} & =\frac{1}{\sqrt{2}}\left(-b_{1}^{\dagger}-i b_{2}^{\dagger}\right)
\end{aligned}
$$

which satisfy

$$
\left[B_{i}, B_{j}^{\dagger}\right]=\delta_{i j}
$$

one can write

$$
\mathcal{H}^{\prime \mathrm{nc}}=\hbar \Omega_{1} B_{1}^{\dagger} B_{1}+\hbar \Omega_{2} B_{2}^{\dagger} B_{2}+\hbar \Omega
$$

with frequencies

$$
\Omega_{1}=\Omega+\frac{K \theta}{2 \hbar}, \quad \Omega_{2}=\Omega-\frac{K \theta}{2 \hbar} .
$$

With (41), we can easily solve the eigenequations

$$
\mathcal{H}^{\prime \mathrm{nc}}\left|n_{1}, n_{2}, \theta\right\rangle=\mathcal{E}_{n_{1}, n_{2}}^{\prime \text { nc }}\left|n_{1}, n_{2}, \theta\right\rangle
$$

and obtain

$$
\left|n_{1}, n_{2}, \theta\right\rangle=\frac{\left(B_{1}^{\dagger}\right)^{n_{1}}\left(B_{2}^{\dagger}\right)^{n_{2}}}{\sqrt{n_{1} ! n_{2} !}}|0,0, \theta\rangle
$$

and the eigenvalues

$$
\mathcal{E}_{n_{1}, n_{2}}^{\prime \mathrm{nc}}=\hbar \Omega_{1} n_{1}+\hbar \Omega_{2} n_{2}+\hbar \Omega .
$$

Projecting $|0,0, \theta\rangle$ on the plane $\left(Y_{1}, Y_{2}\right)$ we find the ground state wave function

$$
\psi_{0}(\vec{Y}, \theta)=\sqrt{\frac{M \Omega}{\pi \hbar}} \exp \left\{-\frac{M \Omega}{2 \hbar}\left(Y_{1}^{2}+Y_{2}^{2}\right)\right\} .
$$


In terms of the $\vec{x}$ representation, it can be written as

$$
\psi_{0}(\vec{x}, \theta)=\sqrt{\frac{M \Omega}{\pi \hbar}} \exp \left\{-\frac{M \Omega}{2 \hbar}\left[e^{\eta}\left(x_{1} \cos \frac{\alpha}{2}-x_{2} \sin \frac{\alpha}{2}\right)^{2}+e^{-\eta}\left(x_{1} \sin \frac{\alpha}{2}+x_{2} \cos \frac{\alpha}{2}\right)^{2}\right]\right\} .
$$

We note that the analysis in the previous section is recovered for $\theta=0$.

\section{Density matrix of the NC system}

Because of its relevance in determining several thermodynamic quantities, it is interesting to calculate the density matrix

$$
\rho\left(\vec{x}, \vec{x}^{\prime}\right)=\psi(\vec{x}) \psi^{*}\left(\vec{x}^{\prime}\right)
$$

of the NC system.

For the ground state wave function (47) the density matrix

$$
\rho_{0}\left(\vec{x}, \vec{x}^{\prime}, \theta\right)=\psi_{0}(\vec{x}, \theta) \psi_{0}^{*}\left(\vec{x}^{\prime}, \theta\right)
$$

can be represented as an integral

$$
\rho_{0}\left(\vec{x}, \vec{x}^{\prime}, \theta\right)=\int \rho_{0}\left(\vec{x}, \vec{x}^{\prime \prime}, \theta\right) \rho_{0}\left(\vec{x}^{\prime \prime}, \vec{x}^{\prime}, \theta\right) d x_{1}^{\prime \prime} d x_{2}^{\prime \prime} .
$$

Tracing $\rho_{0}\left(\vec{x}, \vec{x}^{\prime}, \theta\right)$ over the variable $x_{2}$, the resulting density is

$$
\rho_{0}\left(x_{1}, x_{1}^{\prime}, \theta\right)=\int \psi_{0}\left(x_{1}, x_{2}, \theta\right) \psi_{0}^{*}\left(x_{1}^{\prime}, x_{2}, \theta\right) d x_{2} .
$$

Evaluating this integral, we get

$$
\begin{aligned}
\rho_{0}\left(x_{1}, x_{1}^{\prime}, \theta\right)= & \left(\frac{\Omega M}{\pi \hbar \gamma}\right)^{1 / 2} \exp \left\{\frac{\Omega M}{4 \hbar \gamma}\left(x_{1}+x_{1}^{\prime}\right)^{2} \sin ^{2} \alpha \sinh ^{2} \eta\right\} \\
& \exp \left\{-\frac{\Omega M}{2 \hbar}\left(x_{1}^{2}+x_{1}^{\prime 2}\right)\left(e^{\eta} \cos ^{2} \frac{\alpha}{2}+e^{-\eta} \sin ^{2} \frac{\alpha}{2}\right)\right\}
\end{aligned}
$$

with the abbreviation

$$
\gamma=e^{\eta} \sin ^{2} \frac{\alpha}{2}+e^{-\eta} \cos ^{2} \frac{\alpha}{2}
$$

The diagonal elements are

$$
\rho_{0}\left(x_{1}, x_{1}, \theta\right)=\left(\frac{\Omega M}{\pi \hbar \gamma}\right)^{1 / 2} \exp \left\{-\frac{\Omega M}{\hbar \gamma} x_{1}^{2}\right\} .
$$

We can use (52) to show that the relation

$$
\operatorname{Tr}\left(\rho_{0}(\theta)\right)=1
$$


is satisfied as it should be for a normalized state, where the notion $\rho_{0}(\theta)=\rho_{0}\left(\vec{x}, \vec{x}^{\prime}, \theta\right)$ is used. The trace of $\rho_{0}^{2}(\theta)$ can be obtained by evaluating the integral

$$
\operatorname{Tr}\left(\rho_{0}^{2}(\theta)\right)=\int \rho_{0}\left(x_{1}, x_{1}^{\prime}, \theta\right) \rho_{0}\left(x_{1}^{\prime}, x_{1}, \theta\right) d x_{1}^{\prime} d x_{1}
$$

to end up with

$$
\operatorname{Tr}\left(\rho_{0}^{2}(\theta)\right)=\left(1+\sinh ^{2} \eta \sin ^{2} \alpha\right)^{-\frac{1}{2}} .
$$

Clearly, the traces are $\theta$-independent and therefore they are not affected by the non-commutativity (28). The density matrix obtained by Kim et al. [11] can be recovered by taking the limit $\theta=0$.

\section{Wigner function for the NC system}

Next, we use the wave function (47) to determine the corresponding Wigner function, which in general is defined by 23

$$
W_{n}(\vec{x} ; \vec{p})=\left(\frac{1}{\pi \hbar}\right)^{d} \int e^{-\frac{2 i}{\hbar} \vec{s} \cdot \vec{p}} \psi_{n}^{*}(\vec{x}-\vec{s}) \psi_{n}(\vec{x}+\vec{s}) d^{d} s
$$

for the eigenfunctions $\psi_{n}(\vec{x})$. This definition is based on the operator formulation of quantum mechanics. There is another definition that can be used by introducing the $\hbar$-star-product. These two definitions are equivalent 24. One concrete example was given by Dayi and Kelleyane [25] who evaluated the Wigner function for an electron on the NC plane in the presence of a magnetic field.

For (47) we evaluate the integral

$$
W_{0}(\vec{x} ; \vec{p}, \theta)=\left(\frac{1}{\pi \hbar}\right)^{d} \int e^{-\frac{2 i}{\hbar} \vec{s} \cdot \vec{p}} \psi_{0}^{*}(\vec{x}-\vec{s}, \theta) \psi_{0}(\vec{x}+\vec{s}, \theta) d^{2} s
$$

to get

$$
W_{0}(\vec{x} ; \vec{p}, \theta)=\frac{1}{\pi} f_{0}(\vec{x}, \theta) g_{0}(\vec{p}, \theta)
$$

where the functions $f_{0}(\vec{x}, \theta)$ and $g_{0}(\vec{p}, \theta)$ are

$$
\begin{aligned}
& f_{0}(\vec{x}, \theta)=\sqrt{\frac{M \Omega}{\pi \hbar}} \exp \left\{-\frac{M \Omega}{\hbar}\left[e^{\eta}\left(x_{1} \cos \frac{\alpha}{2}-x_{2} \sin \frac{\alpha}{2}\right)^{2}+e^{-\eta}\left(x_{1} \sin \frac{\alpha}{2}+x_{2} \cos \frac{\alpha}{2}\right)^{2}\right]\right\} \\
& g_{0}(\vec{p}, \theta)=\sqrt{\frac{M \Omega}{\pi \hbar}} \exp \left\{-\frac{M \Omega}{\hbar}\left[e^{-\eta}\left(p_{1} \cos \frac{\alpha}{2}-p_{2} \sin \frac{\alpha}{2}\right)^{2}+e^{\eta}\left(p_{1} \sin \frac{\alpha}{2}+p_{2} \cos \frac{\alpha}{2}\right)^{2}\right]\right\} .
\end{aligned}
$$

Integrating (60) over the variables $x_{2}$ and $p_{2}$, we obtain

$$
\begin{aligned}
& W_{0}\left(x_{1}, p_{1}, \theta\right)=\int W_{0}(\vec{x} ; \vec{p}, \theta) d x_{2} d p_{2}=\frac{M \Omega}{\pi \hbar \sqrt{1+\sinh ^{2} \eta \sin ^{2} \alpha}} \\
& \exp \left\{-\frac{M \Omega}{\hbar(\cosh \eta-\sinh \eta \cos \alpha)} x_{1}^{2}\right\} \exp \left\{-\frac{M \Omega}{\hbar(\cosh \eta+\sinh \eta \cos \alpha)} p_{1}^{2}\right\}
\end{aligned}
$$


Of course at $\theta=0$ we recover the standard Wigner function for this system [1].

One can use $W_{0}\left(x_{1}, p_{1}, \theta\right)$ to verify (55) and (57) by evaluating [9]

$$
\operatorname{Tr}\left(\rho_{0}(\theta)\right)=\int W_{0}(\theta)\left(x_{1}, p_{1}, \theta\right) d x_{1} d p_{1}
$$

as well as

$$
\operatorname{Tr}\left(\rho_{0}^{2}(\theta)\right)=2 \pi \int W_{0}^{2}\left(x_{1}, p_{1}, \theta\right) d x_{1} d p_{1} .
$$

Indeed, these integrals confirm that the traces are $\theta$-independent.

\section{$6 \quad$ Uncertainty relation and entropy on $\mathbb{R}_{\theta}^{2}$}

The expectation value of any operator $A$ is defined by the matrix element

$$
\langle A\rangle=\langle\psi|A| \psi\rangle
$$

where $|\psi\rangle$ is a normalized state. Also it can be expressed as the trace

$$
\langle A\rangle=\operatorname{Tr}(A \rho)
$$

over the physical states.

The uncertainty of the operator $A$ is given by

$$
\Delta A=\sqrt{\left\langle A^{2}\right\rangle-\langle A\rangle^{2}}
$$

Using either (65) or (66) , we calculate the product $\Delta x_{1} \Delta p_{1}$ for the present system on $\mathbb{R}_{\theta}^{2}$

$$
\frac{\Delta x_{1} \Delta p_{1}}{\sqrt{1+\sinh ^{2} \eta \sin ^{2} \alpha}}=\frac{\hbar}{2} \sqrt{1+\left(\frac{M \Omega \theta}{2 \hbar}\right)^{2}}=\frac{\hbar}{2} \sqrt{1+\frac{a^{2}}{1+a^{2}}}
$$

where we have set $a=\frac{m \omega \theta}{2 \hbar}$. This relation can also be obtained by using the Wigner function (62).

For $\theta=0$, we recover the standard result for the present system [1]

$$
\frac{\Delta x_{1} \Delta p_{1}}{\sqrt{1+\sinh ^{2} \eta \sin ^{2} \alpha}}=\frac{\hbar}{2}
$$

For $\theta \longrightarrow \infty$, we obtain

$$
\frac{\Delta x_{1} \Delta p_{1}}{\sqrt{1+\sinh ^{2} \eta \sin ^{2} \alpha}}=\frac{\hbar}{\sqrt{2}}
$$

For small values of $\theta$, (268) can be expanded as

$$
\frac{\Delta x_{1} \Delta p_{1}}{\sqrt{1+\sinh ^{2} \eta \sin ^{2} \alpha}} \approx \frac{\hbar}{2}+\frac{\hbar a^{2}}{4} .
$$




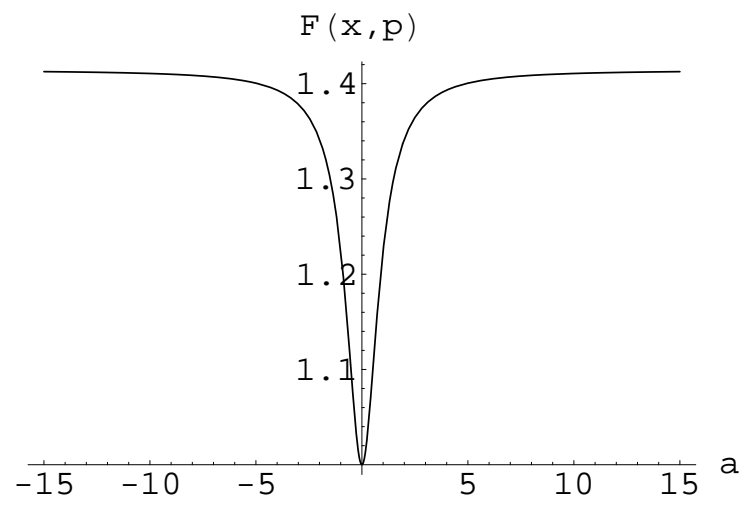

Figure 1: Variation of the uncertainty product $\mathrm{F}(\mathrm{x}, \mathrm{p})=2 \Delta x_{1} \Delta p_{1} / \hbar$ in terms of $a=\frac{m \omega \theta}{2 \hbar}$ for $\eta=0$ or $\alpha=0$.

This shift can be interpreted as a quantum correction to (69). In Figure 1 we show how this relation depends on $\theta$.

Finally, we calculate the entropy [22]

$$
S=-\operatorname{Tr}(\rho \ln \rho)
$$

for the NC system. Using the matrix elements $\left(\rho_{0}(\theta)\right)_{m m}$ of $\rho_{0}\left(x_{1}, x_{1}^{\prime}, \theta\right)$ we determine

$$
S=-\sum_{m}\left(\rho_{0}(\theta)\right)_{m m} \ln \left(\rho_{0}(\theta)\right)_{m m}=2\left[\cosh ^{2} \eta \ln (\cosh \eta)-\sinh ^{2} \eta \ln (\sinh \eta)\right]
$$

which is nothing but that obtained by Kim et al. [11. Thus we conclude that $S$ is not changed by the non-commutativity (28).

\section{$7 \quad$ Particular values of $\alpha$}

To discuss some limits we distinguish two different values of the mixing angle $\alpha$, i.e. 0 and $\frac{\pi}{2}$. In the former case we identify the non-commutativity parameter $\theta$ to the squared magnetic length $l_{B}^{2}$. In this case we show that our system can be linked to an electron on the plane in the presence of the magnetic field $B$.

\subsection{Case $\alpha=\frac{\pi}{2}$}

As we mentioned in our introduction, it is relevant to consider $\alpha=\frac{\pi}{2}$, because this is the case for most physical systems described by two coupled harmonic oscillators. In this limit, we get 
the ground state wave function

$$
\left.\psi_{0}(\vec{x}, \theta)\right|_{\alpha=\frac{\pi}{2}}=\sqrt{\frac{M \Omega}{\pi \hbar}} \exp \left\{-\frac{M \Omega}{2 \hbar}\left[\left(x_{1}^{2}+x_{2}^{2}\right) \cosh \eta-2 x_{1} x_{2} \sinh \eta\right]\right\},
$$

the density matrix

$$
\left.\rho_{0}\left(x_{1}, x_{1}^{\prime}, \theta\right)\right|_{\alpha=\frac{\pi}{2}}=\left(\frac{M \Omega}{\pi \hbar \cosh \eta}\right)^{1 / 2} \exp \left\{\frac{M \Omega}{4 \hbar}\left[\left(x_{1}+x_{1}^{\prime}\right)^{2} \sinh \eta \tanh \eta+\left(x_{1}^{2}+x_{1}^{\prime 2}\right) \cosh \eta\right]\right\}
$$

and the Wigner function

$$
\left.W_{0}(\vec{x}, \vec{p}, \theta)\right|_{\alpha=\frac{\pi}{2}}=\left.\left.\frac{1}{\pi} f_{0}(\vec{x}, \theta)\right|_{\alpha=\frac{\pi}{2}} g_{0}(\vec{p}, \theta)\right|_{\alpha=\frac{\pi}{2}}
$$

where $\left.f_{0}(\vec{x}, \theta)\right|_{\alpha=\frac{\pi}{2}}$ and $\left.g_{0}(\vec{p}, \theta)\right|_{\alpha=\frac{\pi}{2}}$ are

$$
\begin{aligned}
& \left.f_{0}(\vec{x}, \theta)\right|_{\alpha=\frac{\pi}{2}}=\sqrt{\frac{M \Omega}{\pi \hbar}} \exp \left\{-\frac{M \Omega}{\hbar}\left[\left(x_{1}^{2}+x_{2}^{2}\right) \cosh \eta-2 x_{1} x_{2} \sinh \eta\right]\right\} \\
& \left.g_{0}(\vec{p}, \theta)\right|_{\alpha=\frac{\pi}{2}}=\sqrt{\frac{M \Omega}{\pi \hbar}} \exp \left\{-\frac{M \Omega}{\hbar}\left[\left(p_{1}^{2}+p_{2}^{2}\right) \cosh \eta+2 p_{1} p_{2} \sinh \eta\right]\right\} .
\end{aligned}
$$

The uncertainty relation simplifies to

$$
\left.\frac{\Delta x_{1} \Delta p_{1}}{\sqrt{1+\sinh ^{2} \eta}}\right|_{\alpha=\frac{\pi}{2}}=\frac{\hbar}{2} \sqrt{1+\frac{a^{2}}{1+a^{2}}}
$$

\section{2 $\quad$ Case $\alpha=0$}

This case corresponds to the limit $c_{3}=0$. In the standard geometry, the system becomes decoupled. However in the NC plane we still have an effective coupling, which is $\theta$-dependent. Before going on, we note that solving (13) we have two possibilities

$$
e^{\eta_{1}}=\frac{c_{1}}{\sqrt{c_{1} c_{2}}}, \quad e^{\eta_{2}}=\frac{c_{2}}{\sqrt{c_{1} c_{2}}}
$$

The expressions (79) can be simplified by linking the present system to the Hall electron, setting

$$
\omega=\sqrt{\frac{K}{m}} \equiv \frac{B e}{2 m c}, \quad \theta_{H}=4 l_{B}^{2}
$$

where $l_{B}=\sqrt{\frac{\hbar c}{B e}}$ is the magnetic length. Then

$$
c_{1}=c_{2} \equiv \omega \sqrt{m}, \quad e^{\eta_{1}}=e^{\eta_{2}} \equiv 1
$$


In this case, we recover the ground state wave function of the Hall electron

$$
\left.\psi_{0}\left(\vec{x}, \theta_{H}\right)\right|_{\alpha=0}=\sqrt{\frac{m \omega}{\pi \hbar}} \exp \left\{-\frac{m \omega}{2 \hbar}\left(x_{1}^{2}+x_{2}^{2}\right)\right\} .
$$

This leads to the density matrix

$$
\left.\rho_{0}\left(x_{1}, x_{1}^{\prime}, \theta_{H}\right)\right|_{\alpha=0}=\left(\frac{m \omega}{\pi \hbar}\right)^{1 / 2} \exp \left\{\frac{m \omega}{4 \hbar}\left(x_{1}^{2}+x_{1}^{\prime 2}\right)\right\} .
$$

The corresponding Wigner function reads

$$
\left.W_{0}\left(\vec{x}, \vec{p}, \theta_{H}\right)\right|_{\alpha=0}=\left.\left.\frac{1}{\pi} f_{0}\left(\vec{x}, \theta_{H}\right)\right|_{\alpha=0} g_{0}\left(\vec{p}, \theta_{H}\right)\right|_{\alpha=0}
$$

where $\left.f_{0}\left(\vec{x}, \theta_{H}\right)\right|_{\alpha=0}$ and $\left.g_{0}\left(\vec{p}, \theta_{H}\right)\right|_{\alpha=0}$ are

$$
\begin{aligned}
\left.f_{0}\left(\vec{x}, \theta_{H}\right)\right|_{\alpha=0} & =\sqrt{\frac{m \omega}{\pi \hbar}} \exp \left\{-\frac{m \omega}{\hbar}\left(x_{1}^{2}+x_{2}^{2}\right)\right\} \\
\left.g_{0}\left(\vec{p}, \theta_{H}\right)\right|_{\alpha=0} & =\sqrt{\frac{m \omega}{\pi \hbar}} \exp \left\{-\frac{m \omega}{\hbar}\left(p_{1}^{2}+p_{2}^{2}\right)\right\} .
\end{aligned}
$$

\section{Conclusion}

We have investigated quantum mechanically a system of two coupled harmonic oscillators on the non-commutative plane by requiring that the spatial coordinates do not commute and employing the star-product definition. By writing down its NC Hamiltonian and making use of a suitable transformation, i.e. a unitary rotation with the mixing angle $\alpha$, we have ended up with a diagonalized system where the condition (11) for $\alpha$ was taken into account. By solving the eigenequations, the eigenstates and the energy spectrum as well as the ground state wave function are found to depend on the non-commutativity parameter $\theta$ and to coincide for $\theta=0$ with those for the standard case. This was used to determine explicitly the ground state wave function of the NC system before the transformation, which was the starting point of our interest.

Subsequently, we have used the above tools to determine some physical quantities corresponding to the ground state. We have evaluated the corresponding density matrix, which is $\theta$-dependent. However, we have shown that its traces, i.e. $\operatorname{Tr}\left(\rho_{0}(\theta)\right)$ and $\operatorname{Tr}\left(\rho_{0}^{2}(\theta)\right)$, are not affected by the non-commutativity. Also the Wigner function was calculated for the NC system and used to confirm the trace properties. We have explicitly evaluated the uncertainty product and some relevant limits were investigated. By taking small values of $\theta$, we have found that the standard relation was shifted by a term proportional to $\theta^{2}$. This effect was interpreted as 
quantum correction to the normal case. Of course this shift vanishes for $\theta=0$. We have found no $\theta$-dependence for the entropy of the $\mathrm{NC}$ system, it is the same as that for the normal case.

Finally, we have considered some limits of the parameters. In particular, we have studied the case $\alpha=\frac{\pi}{2}$ which is relevant for many physical systems. In another interesting case, $\alpha=0$, the standard system becomes decoupled. However, the NC system retains an effective coupling due to the parameter $\theta$. By identifying $\theta$ to the squared magnetic length, basic features of the Hall system were recovered, in particular its ground state and its Wigner function were given.

This work should be a good starting point to investigate different issues related to the physical systems described by two coupled harmonic oscillators. In particular, it can be used to investigate different models proposed to study many physical problems as mentioned in our introduction, see [5, 6, 8, and $[12-20]$, by deforming the spatial coordinates. We will return to these issues and related matter in future.

\section{Acknowledgments}

AJ's work is supported by Deutsche Forschungsgemeinschaft within the Schwerpunkt "QuantumHall-Effekt". ELEH's work is supported by AB-ICTP within the framework of the associateship scheme.

\section{References}

[1] G. Dunne, R. Jackiw and C. Trugenberger, Phys. Rev. D 41, 661 (1990); G. Dunne and R. Jackiw, Nucl. Phys. (Proc. Suppl.) C 33, 114 (1993).

[2] R.E. Prange and S.M. Girvin (editors), "The Quantum Hall Effect" (New York, Springer 1990).

[3] Y.S. Kim, M.E. Noz, and S.H. Oh, Am. J. Phys. 47, 892 (1979).

[4] Y.S. Kim and M.E. Noz, "Theory and Applications of the Poincaré Group" (Reidel, Dordrecht, 1986).

[5] D. Han, Y.S. Kim, and M.E. Noz, Phys. Lett. A 144, 111 (1989).

[6] Y.S. Kim, Phys. Rev. Lett. 63, 348 (1989).

[7] Y.S. Kim and E.P. Wigner, Phys. Lett. A 147, 343 (1990). 
[8] D. Han, Y.S. Kim, and M.E. Noz, Phys. Rev. A 41, 6233 (1990).

[9] Y.S. Kim and M.E. Noz, "Phase Space Picture of Quantum Mechanics" (World Scientific, Singapore, 1991).

[10] D. Han, Y.S. Kim, and M.E. Noz, J. Math. Phys. 36, 3940 (1995).

[11] D. Han, Y.S. Kim, and M.E. Noz, Am. J. Phys. 67, 61 (1999).

[12] S.S. Schweber, "An Introduction to Relativistic Quantum Field Theory" (Row-Peterson, Elmsford, New York, 1961).

[13] A.L. Fetter and J.D. Walecka, "Quantum Theory of Many Particle Systems" (McGrawHill, New York, 1971).

[14] P.A.M. Dirac, J. Math. Phys. 4, 901 (1963).

[15] C.M. Caves and B.L. Schumaker, Phys. Rev. A 31, 3068 (1985); B.L. Schumaker and C.M. Caves, Phys. Rev. A 31, 3093 (1985).

[16] F. Iachello and S. Oss, Phys. Rev. Lett. 66, 2976 (1991).

[17] H. Umezawa, H. Matsumoto, and M. Tachiki, "Thermo Field Dynamics and Condensed States" (North-Holland, Amsterdam, 1982).

[18] B. Yurke and M. Potasek, Phys. Rev. A 36, 3464 (1987).

[19] A.K. Ekert and P.L. Knight, Am. J. Phys. 57, 692 (1989).

[20] S.M. Barnett and S.J.D. Phoenix, Phys. Rev. A 44, 535 (1991).

[21] A. Connes, "Noncommutative Geometry" (Academic Press, London 1994).

[22] E.P. Wigner and M.M. Yanase, "Information Contents of Distributions", Proc. Nat. Acad. Sciences (U.S.A.) 49, 910 (1963).

[23] E. Wigner, Phys. Rev 40, 749 (1932).

[24] M. Hillery, R.F. O'Connell, M.O. Scully and E.P. Wigner, Phys. Rep. 106, 121 (1984); T. Curtright, D. Fairlie and C. Zachos, Phys. Rev D 58, 025002 (1998); M. Levanda and V. Fleurov, Ann. Phys. 292, 199 (2001); C. Zachos, Deformation Quantization: Quantum Mechanics Lives and Works in Phase-Space, (2001) hep-th/0110114.

[25] Ö.F. Dayi and L.T. Kelleyane, Mod. Phys. Lett. A 17, 1937 (2002). 